\title{
Autoregressive Model Order Estimation Criteria for Monitoring Awareness during Anaesthesia
}

\author{
Nicoletta Nicolaou and Julius Georgiou \\ KIOS Research Centre and Dept. of Electrical and Computer Engineering, \\ University of Cyprus, Kallipoleos 75, \\ 1678 Nicosia, Cyprus \\ \{nicolett,julio\}@ucy.ac.cy
}

\begin{abstract}
This paper investigates the use of autoregressive (AR) model order estimation criteria for monitoring awareness during anaesthesia. The Bayesian Information Criterion (BIC) and the Akaike Information Criterion (AIC) were applied to electroencephalogram (EEG) data from 29 patients, obtained during surgery, to estimate the optimum multivariate AR model order. Maintenance of anaesthesia was achieved with propofol, desflurane or sevoflurane. The optimum orders estimated from the BIC reliably decreased during anaesthetic-induced unconsciousness, as opposed to AIC estimates, and, thus, successfully tracked the loss of awareness. This likely reflects the decrease in the complexity of the brain activity during anaesthesia. In addition, AR order estimates sharply increased for diathermy-contaminated EEG segments. Thus, the BIC could provide a simple and reliable means of identifying awareness during surgery, as well as automatic exclusion of diathermy-contaminated EEG segments.
\end{abstract}

Keywords: anaesthesia, AR model order estimation, awareness, EEG.

\section{Introduction}

Intraoperative awareness is the phenomenon where patients regain consciousness during surgery. It can lead to severe psychological consequences, such as post-traumatic stress disorder, and economic consequences, such as large insurance compensations. The reported incidence of intraoperative awareness ranges from $0.1-0.8 \%$ [1]; however, due to the amnesic effect of certain anaesthetics, some patients have no recollection of regaining awareness, therefore it is likely that the real incidence of awareness is much higher. The problem becomes more serious due to the routine administration of muscle relaxants with anaesthetic agents, thus placing patients who regain awareness in a state of 'awake paralysis'. Several indices have been applied to monitor the depth of hypnosis (for a review see [2]) and some devices are commercially available (e.g. Bispectral Index monitor - BIS). These devices convert some combination of various EEG characteristics into a single number from 0-100 representing the level of hypnosis (100 - 'fully awake', 0 - 'isoelectricity'). Commercial devices suffer from a number of issues, e.g. estimation of the level of hypnosis can be affected by the type 
of anaesthetic or the administration of a muscle relaxant [3, 4, and the large inter-subject variability is not captured by the fixed number that represents the level of hypnosis $[5]$.

In this work we propose a method for monitoring awareness based on tracking the anaesthetic-induced changes in EEG signal complexity. The proposed method is based on the hypothesis that anaesthetics cause a decrease in the optimum order necessary for EEG modelling with autoregressive (AR) models, which are widely used in EEG analysis (e.g. [6 8]). Since estimating the optimum AR model order is non-trivial several criteria have been introduced for this purpose, with two of the most widespread being the Bayesian Information Criterion (BIC) 9] and the Akaike Information Criterion (AIC) 10. The ability of the BIC and the AIC to capture this anaesthetic-induced reduction in EEG complexity is investigated. The choice of these measures for this purpose is motivated by the fact that they can provide a computationally simple method of monitoring awareness, while at the same time addressing some of the issues faced by current monitors.

\section{Methods}

\section{$2.1 \quad$ Dataset}

The data used in the particular study were collected from 29 patients (mean age $42.6 \pm 20.5$; 1 female patient) undergoing routine general surgery at Nicosia General Hospital, Cyprus. The study was approved by the Cyprus National Bioethics Committee and patients gave written informed consent. A detailed description of the dataset and patient exclusion criteria can be found in previous studies (see, for example, [1] ). In summary, anaesthesia was induced with a propofol $(1 \%, 10 \mathrm{mg} / \mathrm{ml})$ bolus. Maintenance of anaesthesia was achieved with (a) intravenous propofol administration (23 patients), or (b) inhalational administration of sevoflurane (4 patients) or desflurane (2 patients). In most patients this was titrated with remifentanil hydrochloride. During induction and/or maintenance some patients received boluses of other drugs, such as neuromuscular blocking agents (cisatracurium, rocuronium, or atracurium) and analgesics, as per surgery requirements.

EEG data were collected using the TruScan32 system (Deymed Diagnostic) at a sampling rate of $256 \mathrm{~Hz}$ using 19 electrodes according to the $10 / 20$ system ( $\mathrm{FCz}$ reference). The data were band-pass filtered at 1-100 Hz. Data recording was performed throughout the entire surgical duration (pre-induction, induction, surgery, recovery of consciousness). The point at which the patients stopped responding verbally to commands by the anaesthetist occurred less than a minute after administration of the anaesthetic bolus, depending on patient characteristics. Recovery of consciousness was defined as the point at which the patient responded to verbal commands or tactile stimuli by the anaesthetist. Patient response was expressed either as voluntary muscular movement in response to a command by the anaesthetist or a verbal response. Throughout the recording, 
time stamps indicating important events, such as anaesthetic induction and recovery of consciousness, were manually inserted in the digital EEG record. These markers were used in subsequent data analysis.

\subsection{Multivariate AR Modelling}

An AR model can be considered as a linear filter, which describes a current value of a time series with $\mathrm{T}$ samples, $\mathbf{X}=\{x(1), x(2), \ldots, x(T)\}$, using weighted information from p past samples:

$$
x(t)=\sum_{i=1}^{p} a_{i} x(t-i)+e_{i}(t)
$$

where $e_{i}$ is normal noise. The standard AR model assumes a 1-dimensional time series. However, in many situations it is desirable that $N$ variables are modelled together. This is particularly useful when this $N$-dimensional information is obtained simultaneously, for example through EEG recordings. For this purpose, the multivariate extension of the AR model (MVAR) can be used [12. In MVAR models information from all $N$-dimensional observations are taken into account in a unified model of order $p$ :

$$
\mathbf{x}(t)=\sum_{i=1}^{p} \mathbf{A}(i) \mathbf{x}(t-i)+\mathbf{E}(t)
$$

where

$$
\mathbf{x}(t)=\sum_{i=1}^{p}\left[\begin{array}{ccc}
a_{11}(i) & \cdots & a_{1 N}(i) \\
\vdots & \ddots & \vdots \\
a_{N 1}(i) & \cdots & a_{N N}(i)
\end{array}\right]\left[\begin{array}{c}
x_{1}(t-i) \\
\vdots \\
x_{N}(t-i)
\end{array}\right]+\left[\begin{array}{c}
e_{1}(t) \\
\vdots \\
e_{N}(t)
\end{array}\right]
$$

\subsection{AR Model Order Estimation}

A number of measures have been developed to estimate the optimum model order. The most widespread are the Bayesian Information Criterion (BIC) [9] and the Akaike Information Criterion (AIC) [10]. These criteria measure the relative goodness of fit of a particular AR model. They provide relative measures of the information loss when the model is applied on the observed data. Given a set of models, the preferred one minimizes the information loss (log-likelihood) estimated from the model order criteria.

The AIC is an information-theoretic measure derived by an approximate minimization of the difference between the true data distribution and the model distribution through the Kullback-Leibler divergence. One way of estimating the AIC is via the residual sum of squares after regression on the data with a model of order $p$,

$$
A I C(p)=\ln \left|\sigma_{e}^{2}\right|+2 \frac{k}{T}
$$


where $k=N \times N \times p$ is the number of parameters estimated for a multivariate model of order $p$ and $N$ dimensions, $\sigma_{e}^{2}$ is the variance of the model residuals, and $T$ is the number of samples. From the definition, the first term of the AIC measures the difference between the log-likelihood of the fitted model and the log-likelihood of the model under consideration. The second term is a penalty term, which penalizes the addition of parameters. The combination of these two terms results in large values of AIC when the model has too few or too many parameters. Thus, the best model is one that provides both a good fit, and that has a minimum number of parameters. This avoids the problem of overfitting. The BIC estimation is very similar to the AIC. However, it differs from the AIC in that it is based on Bayesian formalism and is derived from approximating the Bayes Factor, i.e. the evidence ratios of models. It is defined as:

$$
B I C(p)=\ln \left|\sigma_{e}^{2}\right|+k \ln T
$$

Just as the AIC, the BIC also penalizes free parameters, but it does so more strongly. Previous studies have demonstrated that, in general, the BIC is more consistent in contrast to the AIC 13 . The AIC performs well in relatively small samples, but is inconsistent and its performance does not improve in large samples; this is in contrast to the BIC, which appears to perform more poorly in small samples, but remains consistent and its performance is improved with larger sample sizes.

\subsection{Methodology}

Dimensionality Reduction. The original data space is 19-dimensional (number of electrodes). Fitting a 19-dimensional MVAR model of order $p$ implies estimation of $19 \times 19 \times p$ parameters. The requirement for EEG stationarity does not allow the use of long segments; therefore, such a large number of parameters cannot be estimated reliably. In order to reduce the dimensionality, and subsequently the number of estimated parameters, two approaches were followed:

1. EEG aggregates: five brain areas were defined as the aggregate activity of particular electrodes. These areas were: left frontal (LF: electrodes Fp1, F7, F3, T3, C3), right frontal (RF: Fp2, F8, F4, C4, T4), left posterior (LP: T5, $\mathrm{P} 3, \mathrm{O} 1)$, right posterior ( $\mathrm{RP}: \mathrm{T} 6, \mathrm{P} 4, \mathrm{O} 2)$, and midline (Z: Fz, Cz, Pz). In defining these particular aggregates it was taken into account that frontoposterior interactions appear to play an important role in (un)consciousness. Electrode impedance is measured automatically; thus, electrodes with high impedance were subsequently excluded from aggregates estimation.

2. Frontal EEG: the majority of work in the literature, and also commercially available depth of anaesthesia monitors, base their analysis on mono- or bipolar frontal EEG activity. Here, we also applied the BIC and AIC measures on activity from electrode locations Fp1 and Fp2 only, in order to investigate the reliability of such features. 
Optimum AR Order Estimation. The EEG data of each patient were analysed in non-overlapping windows of $2 \mathrm{~s}$ duration. The window size should be large enough to ensure adequate number of samples for accurate parameter estimation, but also short enough to ensure data stationarity. The choice of 2-s fulfilled both these requirements. For each window the optimum order from a range of $p=2,3, \ldots, 30$ was estimated using the BIC and AIC measures, for both the EEG aggregates and the frontal EEG. For each patient, the differences in the estimated AR orders at the two conditions were tested for statistical significance (ANOVA F-test, $\alpha=0.05$ ).

\section{Results}

Figure 1 shows the resulting BIC (a-b) and AIC (c-d) boxplots for wakefulness and anaesthesia for all patients, for both EEG aggregates (a, c) and frontal EEG $(\mathrm{b}, \mathrm{d})$. Statistical significance was estimated using ANOVA F-test $(\alpha=0.05)$. All differences between wakefulness and anaesthesia were significant. Specifically, for aggregate activity, (1) BIC: $F=3535.01, p=0$, and AIC: $F=5452.03, p=0$. For frontal activity, (1) BIC: $F=616.76, p=0$, and AIC: $F=253.00, p=0$. The median AR orders from the aggregate EEG are (a) BIC: 10 (awake) and 7 (anaesthesia); (b) AIC: 30 (awake) and 14 (anaesthesia). For frontal EEG we obtain (a) BIC: 13 (awake) and 10 (anaesthesia); (b) AIC: 30 (awake) and 30 (anaesthesia). Figure 2 shows the median optimum AR orders estimated for each patient using the BIC (a-b) and AIC (c-d) for wakefulness and anaesthesia. All differences were statistically significant (ANOVA F-test, $\alpha=0.05$ ), except for patient S22 (indicated with an '*' on figure 2). A general observation is the overestimation of the optimum AR orders using the AIC measure.

\section{Discussion}

The optimum AR orders estimated from the BIC using the EEG aggregate activity track the transitions between wakefulness and anaesthesia well. Administration of anaesthesia causes a decrease in the estimated optimum AR orders, which remain stable throughout the entire duration of surgery, and increase again after the patient regains consciousness at the end of surgery. This effect was observed only for the BIC, with more robust estimates obtained from the aggregate EEG. Despite a similar effect observed for the AIC, this was not robust and identification between awake and anaesthetized states was difficult. Despite the large inter-subject differences in the raw EEG activity, the optimum AR orders estimated for anaesthesia and wakefulness did not display large inter-subject variability.

The more robust BIC performance could be explained through a representative example for a randomly chosen patient and EEG segment (fig. 4). The estimated BIC values are in general higher than the AIC values, which is expected if we take into consideration the second term of eqs. 4 and 5. From fig. 4 it can be seen that the changes in the AIC values become negligible for AR models 

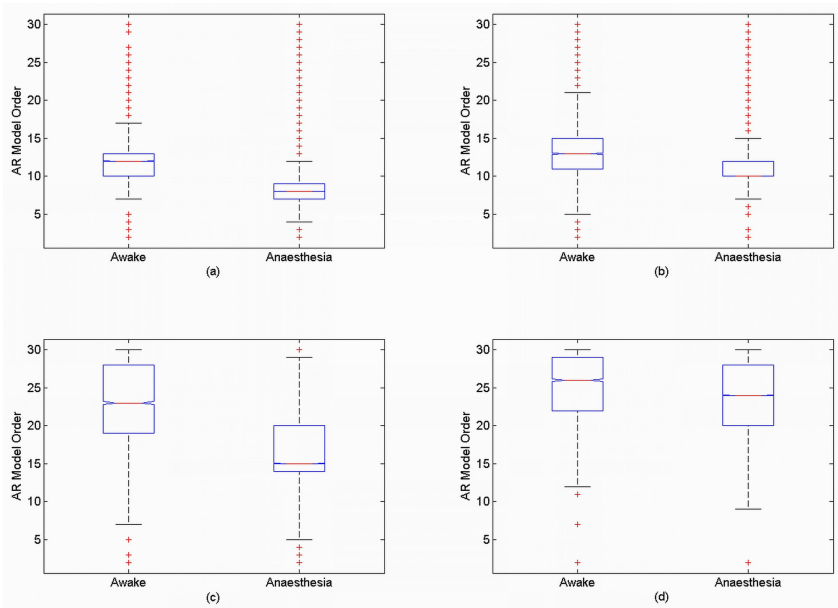

Fig. 1. ANOVA Boxplots over all patients for optimum AR orders estimated during awake and anaesthesia using the BIC (a)-(b), and the AIC (c)-(d). Optimum AR orders in (a) and (c) were estimated from the EEG aggregates, while in (b) and (d) from the frontal EEG. All differences between awake and 'anaesthetized conditions are statistically significant at the $95 \%$ significance level.
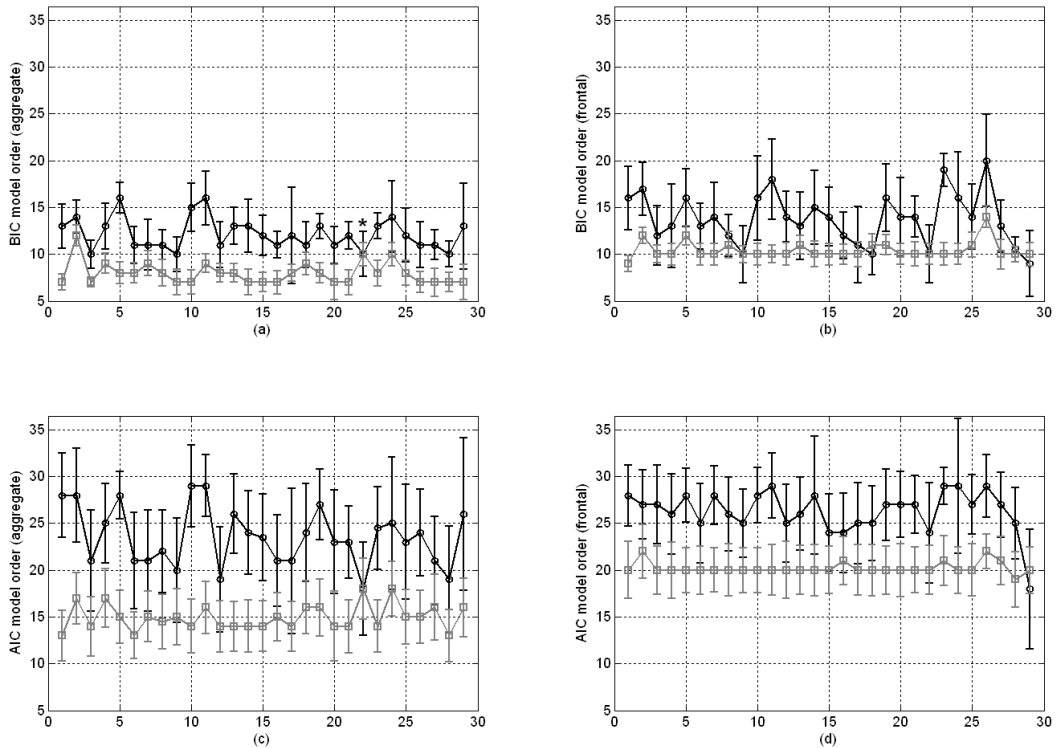

Fig. 2. Median optimum AR orders from BIC (a-b) and AIC (c-d) estimated using EEG aggregate activity (a, c) and frontal activity (b, d), for individual patients. All differences between 'awake' and 'anaesthetized' conditions are statistically significant at the $95 \%$ significance level, except from one difference which is not statistically significant; this is indicated by an '*'. 
A
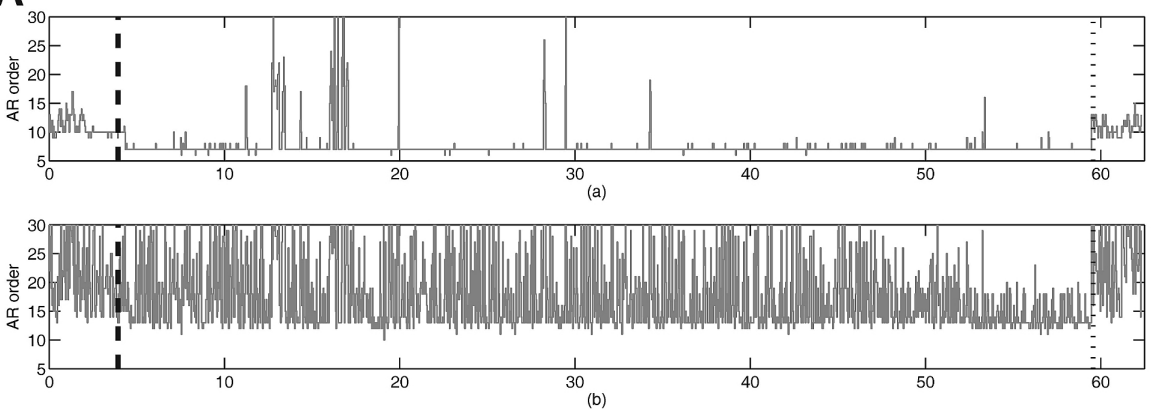

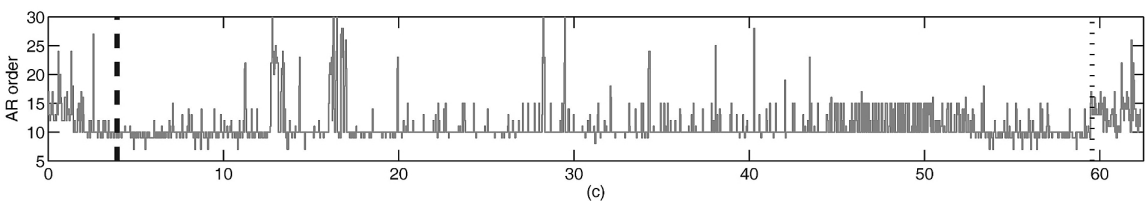

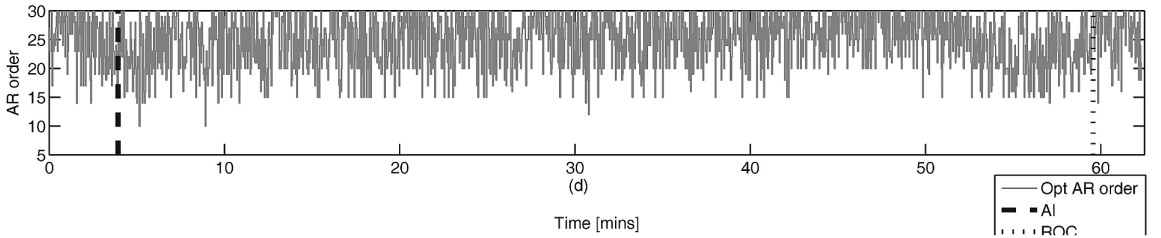

B

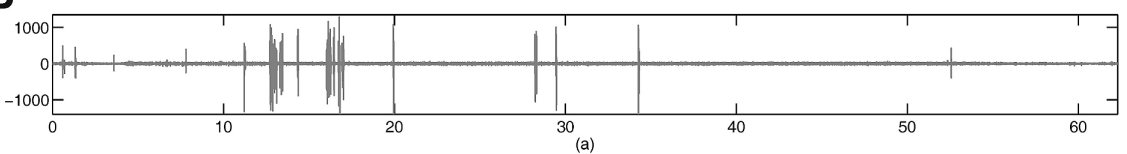

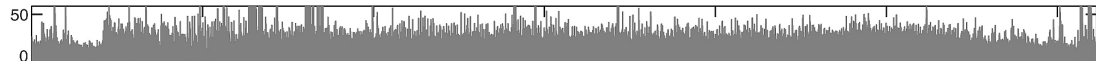

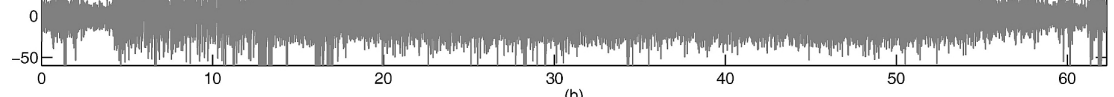

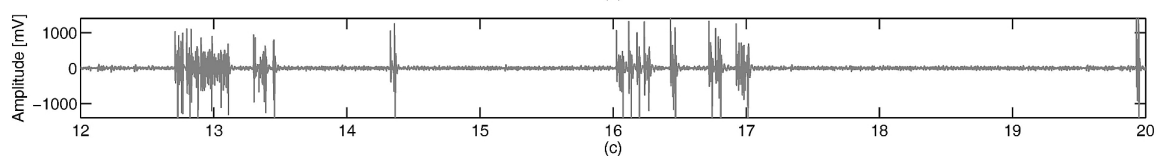

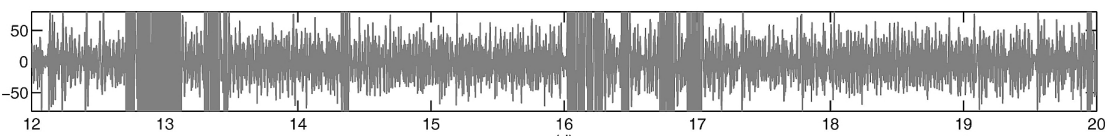

(d)

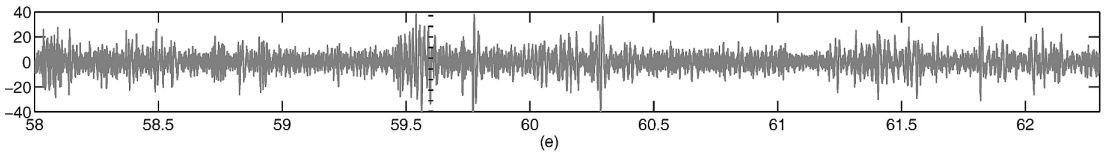

Fig. 2. (Continued.) 

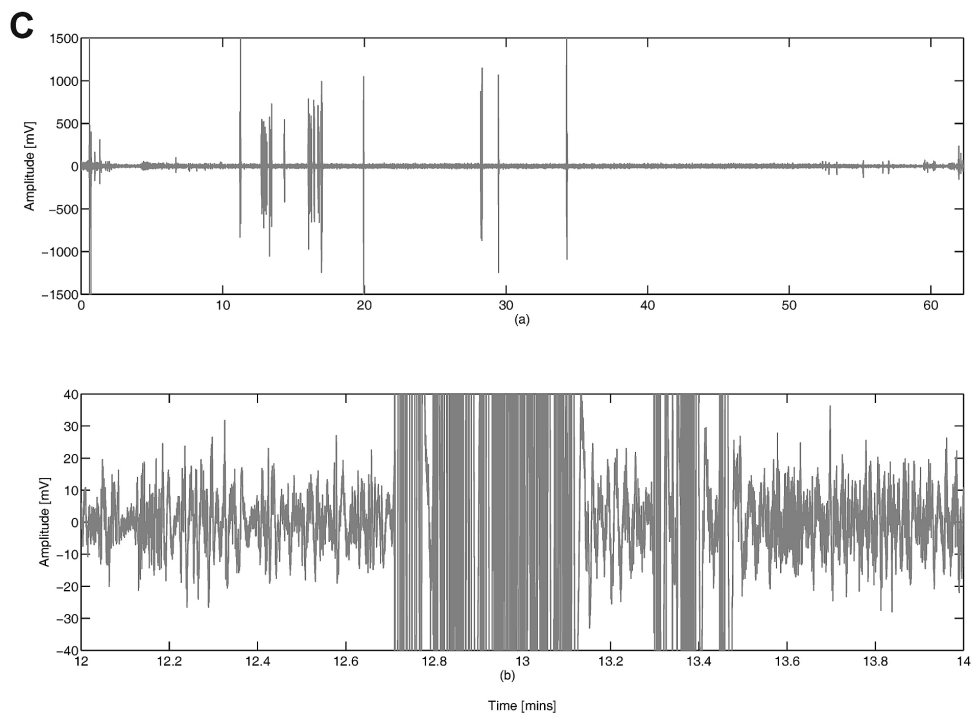

Fig. 3. A: Optimum AR orders for patient S3 throughout entire surgery (maintaince with propofol), estimated by: (a) BIC and (b) AIC from aggregate EEG; and (c) BIC and (d) AIC from frontal EEG. Vertical lines: dashed - induction (AI), dotted - recovery of consciousness (ROC). Sharp peaks in (a) and (c) are caused by diathermy artefacts (see panels B and C for corresponding EEG activity). B: Aggregate EEG for patient S3 throughout surgery (62.3 minutes). Average activity at: (a) left frontal location. High frequency peaks with larger amplitude than the EEG are diathermy artefacts. (b) right frontal location, zoomed in to the amplitude level of the underlying EEG. (c) left posterior at 12-20 min (high diathermy contamination). (d) Right posterior at 12-20 min, zoomed in to EEG amplitude level; and (e) midline at 58-62.3 min (patient recovers consciousness at $56.6 \mathrm{~min}$ ). C: Corresponding frontal EEG activity at (a) Fp1, and (b) Fp2 (zoomed in).

with orders $p>13$. However, the minimum $\mathrm{AIC}$ value is obtained at $p=23$. In contrast, the BIC decreases until a clear minimum value at $p=10$ is reached, after which it increases again. This is a direct result of the BIC estimation, which penalises free parameters more strongly than the AIC. From these observations: (a) the AIC indicates that the goodness of fit is not substantially improved using models of orders $p>13$, therefore an appropriate choice of model order without taking into consideration the absolute minimum $\mathrm{AIC}$ value would be $p=13$; (b) the BIC indicates that the goodness of fit is not substantially improved using models of orders $p>10$, as can be seen by the penalty imposed at such models by the BIC. Therefore, the combination of increasing goodness of fit and larger penalty imposed on the number of free parameters ensures that the BIC attains a clear minimum value at the smallest appropriate order.

The proposed method has several advantages over existing methods or commercial monitors: (1) the estimated features remain stable over the entire surgical 


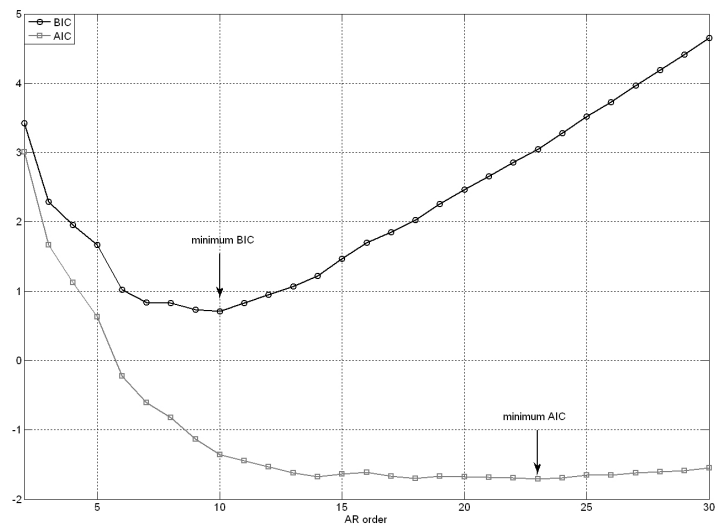

Fig. 4. An example of BIC and AIC values obtained at a randomly chosen EEG segment and a randomly chosen patient. The BIC displays a distinct minimum, as opposed to the AIC, which tends to decrease asymptotically with the model order, resulting into an overestimation of the model order. For this specific example, the optimum AR orders are 10 and 23 as estimated by the BIC and the AIC respectively.

duration; (2) estimations are simple and fast; (3) the features are not affected by the particular anaesthetic protocol; (4) wakefulness/anaesthesia can be identified through simple visual inspection of the estimated features, hence there is no need for conversion to a 'universal' number from 0-100 or the use of a classifier; (5) the estimated AR orders remain stable after anaesthetic administration is switched off, indicating that the AR orders are not simply a reflection of the metabolic decrease of the anaesthetic agent; and (6) diathermy-contaminated EEG segments can be easily identified. In addition, the EEG data were obtained from actual surgery. This allows us to investigate the stability of the estimated AR order patterns and assess the clinical applicability of the proposed method.

\section{Conclusion}

The feasibility of utilizing autoregressive model order criteria for wakefulness / anaesthesia discrimination from EEG activity during surgery was investigated. The Bayesian Information Criterion (BIC) provided a reliable means of tracking the reduction in EEG complexity during anaesthesia through providing lower optimum AR order estimates. This effect can be tracked more reliably when the BIC is applied on aggregate than frontal EEG activity. The same effect was not clearly identifiable with the Akaike Information Criterion (AIC). However, both measures provided AR orders that were statistically different during wakefulness/anaesthesia. The BIC could also be used for automatic identification of diathermy-contaminated EEG segments. Future work will involve (i) investigation of other preprocessing methods for noise removal, e.g. Independent Component Analysis, and (ii) the comparison of the proposed methodology with other 
methods, including other methods previously proposed by the authors (e.g. [1]) and other methods from the literature.

Acknowledgement. The authors would like to thank Dr. Pandelitsa Alexandrou and Dr. Saverios Hourris (Depr. of Anaesthesia, Nicosia General Hospital, Cyprus), the hospital staff and the anonymous volunteers who participated in this study. This work was co-funded by the Republic of Cyprus and the European Regional Development Fund through the Cyprus Research Promotion Foundation ('DESMI 2008'). Grants: New Infrastructure Project/Strategic/0308/26 and DIDAKTOR/DISEK /0308/20. The authors have no conflict of interest.

\section{References}

1. Bruhn, J., Myles, P., Sneyd, R., Struys, M.: Depth of anaesthesia monitoring: what's available, what's validated and what's next? British Journal of Anaesthesia 97(1), 85-94 (2006)

2. Rampil, I.J.: A primer for EEG signal processing in anesthesia. Anesthesiology 89(4), 980-1002 (1998)

3. Dahaba, A.: Different conditions that could result in the bispectral index indicating an incorrect hypnotic state. Anesth. Analg. 101, 765-773 (2005)

4. Russell, I.F.: The Narcotrend "depth of anaesthesia" monitor cannot reliably detect consciousness during general anaesthesia: an investigation using the isolated forearm technique. British Journal of Anaesthesia 96(3), 346-352 (2006)

5. Voss, L., Sleigh, J.: Monitoring consciousness: the current status of EEG-based depth of anaesthesia monitors. Best. Pract. Res. Clin. Anaesthesiol. 21(3), 313-325 (2007)

6. Anderson, C., Stolz, E., Shamsunder, S.: Multivariate autoregressive models for classification of spontaneous electroencephalographic signals during mental tasks. IEEE T Biomed. Eng. 45(3), 277-286 (1998)

7. McFarland, D., Wolpaw, J.: Sensorimotor rhythm-based brain computer interface (BCI): model order selection for autoregressive spectral analysis. J. Neural Eng. 5(2), 155-162 (2008)

8. Franaszczuk, P., Bergey, G.: An autoregressive method for the measurement of synchronization of interictal and ictal EEG signals. Biological Cybernetics 81(1), 3-9 (1999)

9. Schwarz, G.: Estimating the dimension of a model. Annals of Statistics 6(2), 461-464 (1978)

10. Akaike, H.: A new look at the statistical model identification. IEEE Trans Automatic Control 19(6), 716-723 (1974)

11. Nicolaou, N., Houris, S., Alexandrou, P., Georgiou, J.: EEG-based automatic classification of awake versus anesthetized state in general anesthesia using granger causality. PLoS ONE 7(3), e33869 (2012)

12. Penny, W., Harrison, L.: Multivariate autoregressive models. In: Friston, K., Ashburner, J., Kiebel, S., Nichols, T., Penny, W.D. (eds.) Statistical Parametric Mapping: The Analysis of Functional Brain Images, Elsevier, London (2006)

13. Zhang, P.: On the convergence of model selection criteria. Comm. Stat.-Theory Meth. 22, 2765-2775 (1993) 